\title{
Violência e Depressão nas Relações de Intimidade: Estudo entre Jovens Universitários Brasileiros
}

\author{
Violence and Depression in Relationships of Intimacy: \\ study among Brazilian university students \\ Violencia y Depresión en las Relaciones de Intimidad: \\ estudio entre jóvenes universitarios brasileños
}

\author{
Tânia Aldrighi Flake \\ Universidade Anhembi Morumbi, São Paulo, SP, Brasil. \\ E-mail: siberiO1@uol.com.br
}

\begin{abstract}
Resumo
O presente artigo trata de um estudo transversal que faz parte da pesquisa multicêntrica "Estudo Internacional de Violência em Namoro" realizada entre estudantes universitários. e visa apresentar os resultados sobre a relação entre violência na intimidade e depressão, tendo como base as questões de gênero e seus elementos para compreender como se constitui a relação de intimidade. Participaram 362 estudantes de graduação de duas universidades, uma pública e uma privada do estado de São Paulo, sendo 37\% do sexo masculino e 63\% do sexo feminino, e idade média de 20 anos. Para as mulheres, a violência psicológica exclusiva, a violência sexual exclusiva e as violências psicológicas e sexuais concomitantes mostraram-se associadas à depressão; já os fatores de aceitação da violência e das relações de poder só se mostraram associados a situações de violência, mas não se mostraram relacionados à conexão entre depressão e violência. O estudo mostra, de maneira original, que a relação entre depressão e envolvimento em situações de violência pode ocorrer e deve ser melhor investigada.
\end{abstract}

Palavras-chave: violência na família, namoro, depressão, relações interpessoais.

\section{Abstract}

This cross-sectional study belongs to themulticenter survey International Study of Violence in Dating Relationships applied to university students. Its goal is topresent the results on the report between violence in intimacy and depression, based on gender issues and its elements for understanding the constitution of the relationship of intimacy. There were 362 undergraduate students from two universities, one public and one private, of the State of São Paulo, being $37 \%$ male and $63 \%$ female, whose average age was 20 years old. For women, exclusively psychological violence, exclusively sexual violence and psychological and sexual violence together were associated with depression; the factors of violence acceptance and relations of power only presented an association to violence situations, but were not related to the connection between depression and violence. The study shows, in an original way, that this relation between depression and involvement in situations of violence can exist and it should be better investigated.

Keywords: family violence, dating, depression, interpersonal relations. 


\section{Resumen}

El presente artículo es un recorte de la investigación multicéntrica Estudio Internacional de la Violencia en Noviazgo entre jóvenes universitarios, con el objetivo de presentar los resultados del estudio que trató de la relación entre violencia en la intimidad y depresión, teniendo como base conceptual las cuestiones de género y sus elementos para la comprensión de la relación de intimidad. Participaron 362 alumnos de dos universidades, una pública y otra privada, del Estado de São Paulo, siendo el 37\% del sexo masculino y el 63\% del femenino, con edad promedio de 20 años. Para las mujeres, la violencia psicológica exclusiva, la violencia sexual exclusiva y las violencias psicológicas y sexuales simultáneas se mostraron asociadas a la depresión; ya los factores de aceptación de la violencia y de relaciones de poder se mostraron asociados a situaciones de violencia, pero no a la relación entre la depresión y la violencia. El estudio revela que la relación entre depresión e implicación en situaciones de violencia puede ocurrir y se debe investigar mejor.

Palabras-clave: violencia familiar, noviazgo, depresión, relaciones interpersonales.

Já é amplamente reconhecido que a violência está presente no relacionamento entre parceiros íntimos na fase de namoro, parceiros em coabitação ou na relação maritalmente constituída (Barnett, Miller-Perrin, \& Perrin, 1997). Entre 1999 e 2000, a Associação Americana de Psicologia (APA) e a Organização Mundial de Saúde (OMS) apontaram a relevância de estudos sobre a etiologia da violência; na ocasião, a violência entre parceiros íntimos foi identificada como um dos fatores de risco previsíveis de distúrbios mentais (Andrews, Ustun, \& Kessler, 2000). Somando-se a isso, inúmeras pesquisas demonstram que a violência entre parceiros íntimos em situação de conjugalidade tem início muitas vezes na fase de namoro, o que pode determinar um padrão de relacionamento ao longo do ciclo vital (Chan, et al., 2008; Gelles \& Straus, 1988; Mattingly \& Straus, 2008; Straus, 2004a, 2004b, Teten, Ball, Valle, Noonan, \& Rosenbluth, 2009).

Decorrente desta preocupação, foi realizado um estudo principal que propôs três recortes para compreender a violência na intimidade durante a etapa de namoro: um estudo descritivo sobre as prevalências da violência ao longo do relacionamento de namoro e em seus diversos tipos; um segundo estudo, que abordou a violência e os fatores a ela associados; e um terceiro, que avaliou o impacto da violência na saúde mental com base no quadro de depressão. Os dois primeiros recortes já têm seus resultados publicados (Flake, Schraiber, \& Menezes, 2013).
A proposta no presente artigo é apresentar e discutir os resultados do recorte que tratou da relação entre violência na intimidade e a depressão, a fim de avaliar o impacto da violência na saúde mental a partir do quadro de depressão, agravo escolhido, sobretudo em razão dos muitos estudos que tiveram grande repercussão em saúde mental conectada à experiência de violência (Albuquerque, 2013; Andrew, Usturn, \& Kessler, 2000; Lehrer, Buka, Gortmaker,\& Shrier,2006).

A escolha do estudo da relação entre a depressão, como agravo à saúde, e a violência decorre da literatura que considera a depressão como uma das principais consequências da violência no namoro, sobretudo como impacto sobre a saúde mental de quem a sofre. Enquanto a noção de saúde mental engloba uma variedade de tipos de sofrimentos e sintomas mentais, as maiores manifestações na saúde mental daqueles que sofrem violência no namoro estão nos quadros de depressão, de ansiedade e nas somatizações (Coker et al. 2002; Golding, 1999; Kaura \& Lohman, 2007, Sutherland, Sullivan, \& Bybee,2001).

Outros estudos sobre a associação entre a violência física entre parceiros íntimos e a depressão concluem que indivíduos que vivem um relacionamento abusivo sofrem maiores níveis de depressão do que aqueles que não têm relacionamentos violentos. Tais conclusões estão baseadas em estudos, na sua maioria com mulheres vítimas de agressão física por parte de seus parceiros. Os estudos que abordam as diferenças de gênero ainda são insuficientes (Carlson, McNutt, \& Choi2003; Kaura \& Lohman, 2007). 
Dentre aqueles que encontraram a depressão como o quadro mais comumente relatado (Eshelman \& Levendosky, 2012; Goodkind, Gillum, Bybee, \& Sullivan, 2003), Carlson et al. (2003) referem que mulheres vítimas de violência têm quatro vezes mais chances de depressão do que aquelas que não foram vítimas; e na metanálise de mais de 80 estudos também sobre mulheres, feita por Golding (1999), 48\% das vítimas relataram depressão. Essa mesma metanálise conclui que tal associação mostrou-se igualmente importante em estudos populacionais ou com mulheres que estavam em abrigos e as que são usuárias de serviços de emergência de atendimento à violência. Outros estudos com mulheres também identificaram que as que sofreram violência no namoro apresentam taxas variando de 39 (Beach, Jouriles, \& O'Leary, 1985) a 83\% (Campbell, Sullivan, \& Davidson,1995; Kaura \& Lohman, 2007). Consistente com esses resultados, o trabalho de Campbell (2002) constatou que a maioria das mulheres desenvolve os principais sintomas depressivos após o início da agressão no relacionamento.

Apesar de haver um número crescente de estudos que mostram que os homens também são vítimas de violência no namoro, poucos têm examinado diretamente as diferenças das prevalências para homens e mulheres. Alguns estudos sugerem que o efeito da violência no namoro sobre a saúde mental também pode ser prejudicial para os homens (Dye \& Eckhardt 2000; McFarlane et al. 2000; Sabina \& Straus, 2008).

Enquanto esses pesquisadores argumentam que os homens sofrem os efeitos negativos da depressão na saúde mental associados com a vitimização por violência no namoro, a literatura também considera que é mais aceito o agravo na saúde mental das mulheres (Jackson, Cram, \& Seymour, 2000).

Desse modo, o presente estudo buscou verificar, por meio da comparação entre homens e mulheres, a relação entre a depressão e a violência em seus diversos tipos, assim como a influência da recorrência dos atos nessa relação. Para compreender como se constitui a relação de intimidade, adotaram-se como base conceitual as questões de gênero e seus elementos. Tal perspectiva endossa a concepção de gênero como parte da complexidade que se estabelece durante as relações sociais, e das experiências que se sucedem nos contextos diários influenciando o comportamento e os sentimentos dos indivíduos. Tudo isso é remetido ao interior das relações, em que o homem e os atributos masculinos possuem maior poder (Jansen, Watts, Ellsberg, Heise, \& Garcia-Moreno, 2004; Knudson-Martin \& Mahoney, 2009; Schraiber \& D’Oliveira, 2008; Schraiber, D’Oliveira, \& Couto, 2009). Nesse sentido, o gênero foi considerado como uma categoria de análise, uma vez que homens e mulheres refletem as distinções de gênero no nível interpessoal, ao assumirem para si os respectivos papéis normativos de sua cultura.

A conceituação das variáveis violência e depressão, adotadas no presente estudo, deriva das concepções que embasaram o International Dating Violence Study (IDVS), e nortearam a elaboração do instrumento utilizado na coleta de dados (Straus, 2004a, 2004b). Foram consideradas as seguintes definições das variáveis: a violência e seus tipos, e a depressão, ambas utilizadas no presente estudo. A violência física é definida por ações que se manifestam como forma de bater, dar socos, pontapés, ou jogar objeto no parceiro intencionalmente (Bell \& Naugle, 2007; Halpern, Oslak, Young, Martin, \& Kupper, 2001; Sears, Byers, \&Price, 2007). A violência psicológica pode envolver um insulto, uma humilhação, críticas, ameaças, ou fazer um parceiro se sentir culpado ou inferior, e dizer coisas que perturbam ou prejudicam o parceiro (Hamby \& Sugaram 1999; O’leary, 1999). A violência sexual é caracterizada pela intimidação ou coerção deliberada de um parceiro contra o outro para obrigar a participação na relação sexual ou em práticas sexuais não desejadas (Cornelius \& Resseguie, 2007). Depressão são distúrbios somáticos, cognitivos e de humor. A variável referiu-se à presença ou à ausência de comportamentos, sentimentos ou pensamentos atribuídos como possíveis sintomas depressivos, a partir de pelo menos uma resposta positiva às questões que constam (Straus, 2004a). 


\section{Método}

O presente artigo trata sobre um recorte de uma pesquisa que adotou o enfoque quantitativo e consistiu na análise de dados já coletados a partir do estudo epidemiológico de corte transversal denominado Estudo Internacional de Violência no Namoro ( International Dating Violence Study - IDVS). Sua pesquisa empírica foi realizada entre 2002 e 2003, no estado de São Paulo, com uma amostra da população brasileira de universitários, na sua maioria, jovens.

\section{Participantes}

O estudo foi realizado com estudantes universitários maiores de 18 anos de idade que aceitaram o convite para responder a um questionário autopreenchido (Straus et al., 2004a, 2004b). A amostra foi composta por estudantes de graduação matriculados em duas universidades do estado de São Paulo: uma privada localizada na cidade de São Paulo, e a outra pública localizada no vale do Paraíba. Para cada universidade, foi escolhido mais de um curso (ou faculdade). A escolha dos cursos teve como cuidado obter uma distribuição homogênea entre participantes do sexo masculino e feminino, pois alguns tinham uma configuração com predominância masculina, e outros configuração com predominância feminina ou mista. Como resultado, os estudantes que participaram da pesquisa pertenciam aos seguintes cursos de graduação: Administração de Empresas, Comércio Exterior, Educação Física, Jornalismo, Medicina e Psicologia.

O presente estudo valeu-se da mesma definição e captação de sujeitos da pesquisa multipaíses. Inicialmente foram 510 alunos presentes nos espaços da sala de aula, distribuídos entre as duas universidades. Houve um total de 454 respondentes, que correspondeu a $10 \%$ de não resposta. Do total dos 454 respondentes, foram elegíveis 362 universitários (228 mulheres e 134 homens) que se declararam estar ou terem estado em situação de namoro com pelo menos um mês de duração.
A faixa etária dos participantes, que variou de 18 a 40 anos de idade, foi agrupada em duas categorias, uma correspondente ao período dos 18 a 21 anos de idade, e a outra de 22 anos ou mais, tendo como critério serem os intervalos de maior concentração e corresponder à faixa etária do jovem universitário brasileiro, conforme dados do Censo da Educação Superior, do ano de 2010 (INEP, 2012).

\section{Instrumento}

Utilizou-se o instrumento já padronizado e utilizado em muitos estudos: as Escalas de Táticas de Conflito - CTS (Straus, 1979, 2004a, 2004b). Para o estudo maior de parceiros em fase de namoro, uma versão especial foi desenvolvida pelo coordenador do estudo que também é o próprio autor das escalas CTS prévias. Trata-se do questionário denominado "IDVS Questionnaire" que, ao mensurar a violência entre parceiros nas relações de intimidade, tem a teoria do conflito por fundamento teórico, do mesmo modo que as CTS (Adams, 1965; Straus, 1979).

A CTS foi originalmente criada para avaliar conflitos intrafamiliares, com objetivo de determinar as taxas de prevalência de violência física e psicológica no contexto familiar (Straus \& Gelles, 1990). Como já dito, essas escalas foram revistas por um dos autores, Straus, a elaboração do instrumento usado para a violência no namoro resultou em 39 itens agrupados em pares de perguntas e destinados ao participante e ao parceiro, perfazendo um total de 78 questões, com um formato de fácil manuseio e tempo breve de administração e resposta (cerca de 30 minutos). A CTS manteve o agrupamento por par de perguntas em que um aborda o comportamento do participante, e outro o do parceiro, o que é revelado por apenas um dos parceiros da relação (Straus, 2005).

Além da caracterização das violências, o questionário possui caracterizações do perfil pessoal e de relacionamentos do respondente 
(Personal and Relationships Profile-PRP), contando com diversos tópicos, tais como os apresentados no Quadro 1, e os referentes à depressão no Quadro 2.

\section{Quadro 1}

Tópicos do perfil pessoal e de relacionamentos

\begin{tabular}{|l|l|}
\hline Perfil de características pessoais & Escala de relações interpessoais \\
\hline Personalidade Antissocial & Manejo da raiva (AM) \\
Personalidade Borderlin & Distúrbios de Comunicação (DP) \\
Histórico criminal & Conflito (COM) \\
Depressão & Dominação (DOM) \\
Hostilidade de gênero ao homem & Ciúme (JEL) \\
Hostilidade de gênero à mulher & Compromisso com o relacionamento (RD) \\
Histórico de negligência & Relacionamento conflituoso (RC) \\
Desordem de Estresse Pós-Traumático & Autoimagem negativa (NA) \\
Integração Social & Abuso de substância química (SUB) \\
& Condições estressoras (STR) \\
& Adequação social (SD) \\
& Violência socializada (VS) \\
& Aprovação de violência (VA) \\
& Histórico de abuso sexual (SAH) \\
\hline
\end{tabular}

\section{Quadro 2}

Questões referentes à variável depressão

\section{Depressão}

Eu tenho prazer com o dia-a-dia da minha vida.

Frequentemente, eu acordo me sentindo muito bem.

Minha vida geralmente transcorre muito bem.

Frequentemente eu estou de bom humor.

Eu estou tão triste, que às vezes eu pergunto a mim mesmo por que continuo a viver.

Eu penso que acontecerão boas coisas para mim no futuro.

Coisas terríveis aconteceram comigo que me deixaram indefesa(o) e horrorizada(a)

Eu tenho pensado em me matar.

As variáveis independentes neste estudo foram as já definidas e apresentadas nas duas dimensões de estudo preliminares: sexo, faixa etária, coabitação, tempo de namoro, ter vida sexual, ciúme, histórico de abuso na infância, ideia negativa de gênero, aprovação de violência e uso de substâncias psicoativas (Flake et al., 2013; Straus, 2004a). A variável violência sofrida foi categorizada como violência física, violência exclusivamente psicológica, violência psicológica, violência sexual exclusiva e a combinação de violência física e sexual. Além disso, a variável violência sofrida foi categorizada de acordo com a repetitividade dos tipos de violência.

\section{Procedimentos}

Os procedimentos adotados foram os previstos pelo IDVS, de forma a estar de acordo com os preceitos éticos de pesquisa em seres humanos. A pesquisa foi aprovada pelo Comitê de Ética em Pesquisa da instituição que a sediou pesquisa (15/02/ 2002, Protocolo no 92/09/2001) e da que sediou o presente estudo (17/11/ 2001, Protocolo $n^{\circ} 293 / 10$ ). 
Oquestionário foi distribuído pelos pesquisadores responsáveis em cada país no espaço das salas de aula, em universidades escolhidas por conveniência, em todos os 37 países participantes. O critério de escolha das universidades foi ser um centro oficial e reconhecido de ensino superior em cada país, estar em funcionamento regular, identificar-se ao tipo comum de centros universitários do país e permitir o trabalho de campo.

\section{Análise de dados}

Para o objetivo de estudar a relação entre depressão e violência, foi selecionada a variável depressão como um recorte para o estudo da relação entre violência e saúde mental, por ser este um dos quadros mais importantes apontados pela literatura que estuda a violência interpessoal. $\mathrm{Na}$ análise, as associações entre os eventos relacionados à depressão e à violência sofrida foram consideradas como variáveis de ajustes, tais como ter vida sexual, faixa etária, coabitação, relacionamento atual, tempo de namoro, ciúme, histórico de violência familiar, aceitação da violência, ciúme, ideia negativa de gênero masculino e ideia negativa de gênero feminino.

Para estimar as razões de prevalências (RP) com intervalos de $95 \%$ de confiança (IC), utilizou-se a análise de regressão de Poisson com estimativa de variância robusta (Coutinho, Scazufca, \&Menezes, 2008). Inicialmente, realizou-se o modelo de regressão univariada, diferenciando-se as categorias em violência sofrida na população de estudo, violência perpetrada na população de estudo; violência sofrida para homens e mulheres, e violência perpetrada para homens e mulheres, adotandose os níveis de significância de $20 \%$. Para compor o modelo de regressão multivariada, foram consideradas todas as variáveis associadas ao evento violência sofrida e à violência perpetrada com valores de $p<0,20$. Permaneceram no modelo final as variáveis independentes que se mantiveram associadas ao desfecho, após o ajuste para as referidas variáveis discriminadas. Para aceitação das associações investigadas no modelo final, adotou-se o valor de $p<0,05$. O software estatístico usado para este estudo foi o Stata versão 10.0.

Para a estimativa da associação entre violência sofrida e depressão, foi realizado modelo de Poisson com estimativa de variância robusta do tipo confirmatório. O modelo final foi ajustado pelas demais variáveis que apresentaram $p<0,20$ na análise univariada. O nível de significância adotado foi de $5 \%$.

\section{Resultados}

Para a exposição aos episódios de violência, a variável foi categorizada por tipo de violência, discriminada em violência exclusivamente psicológica, violência física, violência sexual sem a física, e violência física e sexual concomitantes. Já para a recorrência dos episódios de violência, estas foram categorizadas como não ter sofrido nenhuma vez violência, ter sofrido uma vez qualquer violência, e ter sofrido mais de uma vez qualquer violência.
Na tabela 1, encontra-se a análise univariada da associação entre depressão e tipos de violência para mulheres e homens. As categorias ter sofrido violência do tipo físico, do tipo sexual sem o físico e do tipo sexual e físico concomitantes mostraramse, para as mulheres, associadas à depressão, enquanto que para os homens as categorias ter sofrido violência sexual sem a física e ter sofrido violência física e sexual concomitantes mostraramse associadas à depressão. 
Tabela 1

Análise univariada da associação entre a depressão e os tipos de violência entre jovens universitários. São Paulo, 2002.

\begin{tabular}{|c|c|c|c|c|c|c|c|c|}
\hline Variáveis & \%(N) & $\mathbf{R P}^{*}$ & IC(95\%)** & $p$ & \%(N) & RP* & IC(95\%)** & $p$ \\
\hline & \multicolumn{4}{|c|}{ Mulheres } & \multicolumn{4}{|c|}{ Homens } \\
\hline $\begin{array}{l}\text { Violência psicológica } \\
\text { exclusiva }\end{array}$ & $57,2 \%(32)$ & 1,18 & $0,82-1,69$ & 0,349 & $57,1 \%(20)$ & 0,81 & $0,56-1,18$ & 0,263 \\
\hline Violência física & $73,1 \%(19)$ & 1,51 & $1,05-2,18$ & 0,024 & $53,3 \%(8)$ & 0,76 & $0,45-1,29$ & 0,319 \\
\hline Violência sexual sem física & $61,9 \%(39)$ & 1,28 & $0,91-1,80$ & 0,146 & $50,0 \%(13)$ & 0,71 & $0,45-1,12$ & 0,145 \\
\hline $\begin{array}{l}\text { Violência física e sexual } \\
\text { concomitantes }\end{array}$ & $75,9 \%(22)$ & 1,57 & $1,11-2,22$ & 0,010 & $45,5 \%(10)$ & 0,65 & $0,39-1,08$ & 0,102 \\
\hline
\end{tabular}

Nota. ${ }^{*} \mathrm{RP}$ - Razões de prevalência; ${ }^{* *} \mathrm{IC}$ - Intervalo de Confiança

$\mathrm{Na}$ tabela 2, apresenta-se o modelo confirmatório final em que são considerados os tipos de violência e a associação com depressão. Para as mulheres, ter sofrido violência física exclusiva e ter sofrido violência física e sexual concomitantes mantiveram-se associadas à depressão. Ter sofrido violência sexual sem a física perde associação ao se incluir a variável uso de substância psicoativa.

Para os homens, ter sofrido violência sexual sem a física e ter sofrido violência física e sexual perde a associação ao se controlar por tempo de namoro. Isso talvez se deva ao fato de os homens com depressão terem um tempo de duração de namoro menor, e com isso um tempo menor de exposição a sofrer violência no namoro.

\section{Tabela 2}

Modelo confirmatório da associação entre a depressão e os tipos de violência entre mulheres universitárias. São Paulo, 2002.

\begin{tabular}{lccc}
\hline Variáveis & RP* & IC(95\%)** & M \\
\hline & \multicolumn{3}{c}{0,263} \\
Violência psicológica exclusiva & 1,23 & $0,85-1,76$ & 0,026 \\
Violência física exclusiva & 1,52 & $1,05-2,22$ & 0,106 \\
Violência sexual sem a física & 1,34 & $0,93-1,92$ & 0,008 \\
Violência sexual e física concomitante & 1,61 & $1,13-2,29$ & \\
\hline
\end{tabular}

Nota. Modelo ajustado por ter vida sexual, relacionamento atual, tempo de namoro e violência na infância. *RP - Razões de prevalência; ${ }^{* *} \mathrm{IC}$ - Intervalo de Confiança

$\mathrm{Na}$ tabela 3 , encontra-se a análise univariada da associação entre a recorrência de episódios de violência e a depressão para mulheres e homens. Mostraram-se associadas à depressão as categorias ter sofrido violência uma vez e ter sofrido mais de uma vez para as mulheres, enquanto que para os homens ter sofrido violência mais de uma vez mostrou-se associado.

Nas tabelas 4 e 5, é considerado o modelo final confirmatório para a recorrência de episódios de violência sofrida; tanto para os homens quanto para as mulheres não houve nenhuma associação. 
Tabela 3

Análise univariada para a associação entre a depressão e a recorrência de violência entre jovens universitários. São Paulo, 2002.

\begin{tabular}{lcccccccc}
\hline Variáveis & $\mathbf{\% ( N )}$ & $\mathbf{R P}^{*}$ & $\mathbf{I C}(\mathbf{9 5 \%})^{* *}$ & $\boldsymbol{P}$ & $\mathbf{\%}(\mathbf{N})$ & RP* $^{*}$ & IC(95\%)** & $\boldsymbol{P}$ \\
\hline \multicolumn{4}{c}{ Mulheres } \\
Nenhuma violência sofrida & $43,9 \%(18)$ & 1,03 & $0.95-1,12$ & 0,411 & $68,0 \%(17)$ & 0,90 & $0,78-1,03$ & 0,152 \\
Sofreu violência uma vez & $61,8 \%(21)$ & 1,40 & $0,90-2,17$ & 0,126 & $61,1 \%(11)$ & 0,89 & $0,56-1,42$ & 0,648 \\
Sofreu violência mais de uma vez & $65,8 \%(52)$ & 1,49 & $1.02-2,19$ & 0,038 & $50,0 \%(19)$ & 0,73 & $0,48-1,11$ & 0,150 \\
\hline
\end{tabular}

Nota. Modelo ajustado por ter vida sexual, relacionamento atual, tempo de namoro e violência na infância; ideia negativa de comportamento masculino e uso de substância psicoativa (álcool e drogas associados). ${ }^{*} \mathrm{RP}$ - Razões de prevalência; ${ }^{* *} \mathrm{IC}-$ Intervalo de Confiança.

Tabela 4

Modelo confirmatório da associação entre a depressão e a recorrência de violência entre mulheres universitárias. São Paulo, 2002.

\begin{tabular}{lccc}
\hline Variáveis & RP* & IC (95\%)** & P \\
\hline Sofreu violência uma vez & \multicolumn{3}{c}{ Mulheres } \\
Sofreu violência mais de uma vez & 0,72 & $0,44-1,19$ & 0,213 \\
\hline
\end{tabular}

Nota. Modelo ajustado por ter vida sexual, relacionamento atual, tempo de namoro, violência na infância e uso de substância psicoativa (álcool e drogas associadas). *RP - Razões de prevalência; ${ }^{* *} \mathrm{IC}$ - Intervalo de Confiança.

Tabela 5.

Modelo confirmatório da associação entre a depressão e a recorrência de violência entre homens universitários. São Paulo, 2002.

\begin{tabular}{lccc}
\hline Variáveis & RP* & IC (95\%)** & P \\
\hline & Homens & & \\
\hline Sofreu violência uma vez & 1,32 & $0,86-2,04$ & 0,198 \\
Sofreu violência mais de uma vez & 1,42 & $0,96-2,10$ & 0,074 \\
\hline
\end{tabular}

Nota. Modelo ajustado por tempo de namoro. ${ }^{*} \mathrm{RP}$ - Razões de prevalência; ${ }^{* *} \mathrm{IC}$ - Intervalo de Confiança.

\section{Discussão}

A presente análise sobre a depressão relacionada à violência no namoro é pioneira no Brasil, além de especialmente apresentar informações sobre o impacto na saúde mental tanto para mulheres quanto para homens. O estudo de Sabina e Straus (2008), vinculado ao estudo maior internacional (IDVS) do qual foi extraído o presente banco de dados, teve como foco a análise da relação das violências e os transtornos mentais. Esse estudo apresenta os resultados de cada um dos tipos de violência e a associação com sintomas depressivos, como também amplia o estudo das sobreposições da violência, denominada pelos autores de polivitimização, tendo como justificativa o fato de aproximadamente $75 \%$ das populações estudadas terem experimentado dois ou mais tipos de agressão no relacionamento (Coker etal.2002; Sabina \&Straus, 2008).

Sabina e Straus (2008) referem ter encontrado somente dois estudos, de Coker et al. (2002) e de Basile, Arias, Desai, e Thompson (2004), que realizaram a análise considerando as diversas combinações dos tipos de violência sofrida e a relação com sintomas depressivos. Ressalta-se que ambos os estudos não tiveram a relação de namoro como exclusiva, mas sim abarcaram as diversas combinações de relacionamento por parceiro íntimo.

Tal como nesses estudos, no presente caso considerou-se cada um dos tipos de violência como 
também as sobreposições entre eles, classificadas em violência psicológica exclusiva, violência física exclusiva, violência sexual (sem a violência física) e as violências física e sexual concomitantes. Quando consideradas as sobreposições, os resultados mostraram-se um pouco diferentes dos encontrados no estudo de Sabina e Straus (2008), em que os sintomas depressivos se mostraram associados com sofrer violência psicológica para homens, enquanto que para as mulheres, com sofrer violência do tipo físico e psicológico.

No estudo brasileiro, não houve nenhuma associação para os homens; porém, para as mulheres, a violência psicológica exclusiva, a violência sexual exclusiva e as violências psicológicas e sexuais concomitantes mostraramse associadas à depressão.

Os resultados do modelo confirmatório utilizado nesse estudo são bem semelhantes, pois se mostraram associadas à depressão para as mulheres ter sofrido as violências física exclusiva e física e sexual concomitantes. Para os homens, também não se encontrou nenhuma associação com a depressão, apesar de que na análise univariada, na presente pesquisa, mostraram-se associadas as variáveis ter sofrido violência sexual sem a física e ter sofrido violência física e sexual concomitantes.

O estudo de Amanor-Boadu et al. (2011) avaliou a diferença entre jovens universitários e o impacto da violência, no namoro, nos transtornos mentais (destacando a depressão), problemas com álcool e nível de satisfação no relacionamento, com especial atenção à violência física. Os resultados indicam que sofrer violência física, ainda que moderada, esteve associada ao aumento dos níveis de depressão, enquanto a violência grave esteve associada ao aumento de sofrer danos físicos. Os resultados ainda indicaram que sofrer violência física, tanto para homens com para mulheres, implica consequências para a saúde mental como um todo.

Ressalta-se que, no presente estudo, a violência física foi a de maior destaque na associação da depressão, fato consonante com o estudo de Amanor-Boadu et al. (2011); porém, diferentemente do encontrado por estes pesquisadores, essa associação só se manteve para as mulheres. Alguns estudos também apresentaram as sobreposições das violências diferenciando-as em moderadas e graves, e demonstraram que as do tipo grave aumentam o risco de sintomas depressivos (Basile et al. 2004; Coker et al.,2002; Sabina \& Straus, 2008), em contraste com o estudo de Amanor-Boadu et al. (2011).

O presente estudo não considerou a gravidade, mas esta pode estar representada nos resultados pelo fato de não só a violência física exclusiva como também a concomitante com a sexual se mostraram associadas à depressão para as mulheres. Tais dados revelam a importância da análise da concomitância dos tipos de atos ocorridos nas violências, o que deve ser bem considerado nos serviços de saúde. Também chama atenção o fato de que aqueles que já sofrem violência física e sexual têm maior probabilidade de procurar ajuda e serem diagnosticados. Adicionalmente, deve-se pensar também na perspectiva dos agressores e sua superposição com a vitimização.

No contraste dos resultados entre homens e mulheres, pode-se dizer que se constata uma reafirmação de que as consequências da vitimização no namoro para as mulheres são mais expressivas; para os homens, é importante se examinarem com maior detalhamento as especificidades para a questão do ponto de vista masculino. Neste, cabe lembrar que, tendo por base a teoria de gênero, para os homens o envolvimento em situações de violência, além de extremamente superpostas à vitimização com a perpetração, pode ainda não representar sofrimentos mentais, e sim reafirmações de identidades culturalmente valiosas, não suscitando sentimentos negativos.

Campbell et al. (1995), por exemplo, afirmam que o agravo à saúde está relacionado à vitimização, enquanto os problemas de saúde mental de perpetradores são tipicamente vistos como causas ou desencadeantes do comportamento violento, mas não consequências (Bland\&Orn, 1986; Dinwiddie, 1992).

No tocante à recorrência da violência, existem poucos estudos sobre esta temática, e estes 
focalizam as mulheres. Eshelman e Levendosky (2012) mostram que, com o aumento da frequência dos episódios de violência, há um aumento nos sintomas depressivos, como também no número e na extensão dos ferimentos. Outro estudo (DeJonghe\&Levendosky, 2008) apresenta a relação entre a repetitividade e o tempo de exposição à violência, como os piores fatores para problemas de saúde mental entre as mulheres.

As variáveis estudadas no presente estudo foram não sofreu violência, sofreu violência uma vez e sofreu violência mais de uma vez. Na análise univariada, ter sofrido violência uma vez e ter sofrido mais de uma vez mostraram-se associadas à depressão para as mulheres, enquanto para os homens somente ter sofrido violência mais de uma vez. No modelo confirmatório, as recorrências não se mostraram associadas nem para os homens nem para as mulheres.

Uma primeira conclusão em relação a resultados apresentados é que a repetividade deve ser mais explorada em estudos futuros, sendo bastante plausível que esteja relacionada não só ao surgimento de sofrimento mental, como também à gravidade deste, tal como apontam estudos de violência por parceiro íntimo na conjugalidade (Carlson et al. 2003; Clements, Speck, Crane, \& Faulkner, 2004; Goodkind et al. 2003; Howard \& Wang, 2003).

Quanto a outros fatores que reforçariam a ligação entre violência por parceiro íntimo e depressão, a metanálise de Golding (1999) indicou a aceitação da violência no namoro como uma variável importante, assim como Jackson et al. (2000); e Campbell et al. (1995) apontam as relações de poder como um possível fator de mediação entre a violência e a depressão.

Em uma variedade de populações, a depressão está relacionada com a falta de controle e o poder sobre a vida (Mirowsky\&Ross, 1989). Os pesquisadores têm apresentado a relação entre depressão em mulheres, impotência e falta de controle dentro de relações íntimas (Campbell et al., 1995; Hume, 2008). Uma possívelexplicação para essa relação é que a violência dentro de relações resulta em desequilíbrios de poder entre a vítima e o perpetrador; e os sentimentos de impotência, causados por um relacionamento abusivo, em longo prazo são preditivos para depressão em mulheres mais do que a própria violência (Campbell et al., 1995), o que indica que a ligação entre a violência e depressão pode ser decorrente da questão de poder.

Nesse estudo, os fatores 'aceitação da violência' como conduta social e 'relações de poder' apenas se mostraram associados às situações de violência, e para as mulheres no recorte já apresentado no estudo dos fatores associados. No entanto, não se mostraram relacionados à ligação entre depressão e violência neste modelo confirmatório.

Um aspecto de destaque apontado por Barnett (2000) é o fato de as mulheres com histórico de vulnerabilidade à depressão terem maior probabilidade de responder ao estressor da violência na intimidade com depressão, ou contribuir para uma diminuição da probabilidade de sair do relacionamento. Para Leher et al. (2006), os sintomas depressivos estão associados ao risco de sofrer violência nos relacionamentos futuros, independente de fatores como a violência no namoro anterior ou histórico de violência na infância. Os autores também encontraram que o risco para sofrer violência no namoro aumentava conforme aumentavam os sintomas depressivos.

Essa relação também pode ser pensada a partir dos resultados obtidos no presente estudo, em razão de o mesmo ser do tipo transversal e não apresentar o controle da temporalidade entre os eventos. Essa relação pode ser entendida, ao menos, para a relação entre a violência atual, incluída na medida da violência na vida, e a depressão atual. Por outro lado, essas associações ficam mais fracas, pois a relação pode perder força com o passar do tempo, desde a exposição à situação de violência. Não obstante, o que este estudo mostra de forma original é que essa relação entre a depressão e o envolvimento em situações de violência pode ocorrer e deve ser mais bem investigada em pesquisas e em atendimentos nos serviços para mulheres e também para homens. 


\section{Considerações Finais}

Os estudos sobre mulheres, e em geral com a situação de conjugalidade, apontam sempre para a forte associação entre sofrer a violência e ter sintomas de depressão ou especialmente ideação e tentativa de suicídio (DeVries et al., 2011), muito embora em alguns estudos, como na presente pesquisa, o desenho transversal impede a clara linha da temporalidade nas relações causais. Não obstante, é valido o dado de que há forte associação entre esses eventos (sofrer violência e ter sintomas de depressão ou ideação/tentativa de suicídio), em qualquer relação sequencial, associação esta encontrada para as mulheres no presente estudo.

Já para os homens essa associação não foi encontrada. Em um estudo paulista com homens em situação de conjugalidade (Albuquerque, 2013), os dados demonstram associação entre sofrer violência e ter sofrimento mental, a qual se modifica quando ajustada para homens que são também agressores. O autor do estudo, porém, chama a atenção para a pequena amostra de homens que seriam apenas agressores. Essa diferenciação entre vítimas e agressores não pode ser feita no presente estudo dada a grande superposição dessas condições, mas não há dúvida de que temos aqui uma questão a ser melhor pesquisada: uma outra nova hipótese a ser estudada, agora mostrando sentido diverso do sofrimento mental para homens e para mulheres, implicado em situações de violência em parcerias íntimas afetivo-sexuais.

Esse sentido diverso também pode ser interpretado em conformidade com a teoria de gênero aqui utilizada: o comportamento violento parte da educação dos meninos e sua socialização nos referenciais de masculinidade são hegemônicos culturalmente, bem como parte da aceitação da violência como norma social (Couto \&Schraiber, 2005; Gomes, 2010). O significado distinto desse comportamento para homens relativamente às mulheres com sofrimento mental, portanto, pode resultar em relação distinta com o desenvolvimento de sofrimento mental.

Os achados deste estudo têm implicações tanto para a prática clínica quanto para a pesquisa futura. Para ambos os contextos, deve ser de particular importância olhar para outras formas abusivas de relacionamento, além das do tipo físico, uma vez que estas também podem contribuir indiretamente para a depressão, aumentando a exposição a outros estressores. Além disso, não se deve esquecer que a população da qual tratamos nesta pesquisa refere-se a jovens adultos que ainda se encontram na fase de exploração das relações afetivas, em que o jovem experimenta uma quantidade considerável de mudanças e instabilidade, enquanto a experimentação de várias possibilidades de amar e trabalhar caminha no sentido da criação de uma estrutura de vida.

Por fim, destaca-se que a pesquisa sobre violência nas relações de intimidade concentrada numa etapa anterior à da conjugalidade ter recebido maior atenção dos pesquisadores devido às consequências nefastas dessa violência, na relação atual e como um precursor para a violência futura. No entanto, mesmo com vários estudos demonstrando a alta prevalência da violência, pouca atenção tem sido dada para o desenvolvimento de teorias específicas para a violência no namoro, dos agravos na saúde mental dos envolvidos neste tipo de relação e de avaliação da relação entre a violência no namoro e a violência casamento.

Assim, conhecer o padrão da violência no namoro com os impactos na saúde mental de jovens também pode contribuir para a compreensão de importantes questões na violência que acontece no casamento, principalmente porque esta última tradicionalmente acontece no espaço privado dos lares, ao passo que muitas das agressões do namoro acontecem no espaço público da escola. 


\section{Referências}

Albuquerque, F. P., Barros, C. R. S., \&Schraiber, L. B. (2013). Violência e sofrimento mental em homens na atenção primária à saúde. Revista de Saúde Pública, 47(3), 531539. doi: 10.1590/S0034-8910.2013047004324

Amanor-Boadu, Y., Stith, S. M., Miller, M. S., Cook, J., Allen, L., \&Gorzek, M. (2011). Impact of dating violence on male and female college students. Partner Abuse, 2(3), 323-343. doi: 10.1891/1946-6560.2.3.323

Andrew, G., Ustun, T. B., \&Kessler, R. C. (2000). Shutting the stable door: Identifying avertable risk factors for mental disorders. Paper presented at the WHO Burden of Disease Meeting. Auckland: New Zealand.

Barnett, O. W. (2000). Why battered women do not leave, part 1. Trauma, Violence, \& Abuse, 1(4), 343-372. doi: 10.1177/1524838000001004003

Basile, K. C., Arias, I., Desai, S., \&Thompson, M. P. (2004). The differential association of intimate partner physical, sexual, psychological, and stalking violence and posttraumatic stress symptoms in a nationally representative sample of women. Journal of Traumatic Stress, 17(5), 413-421. doi: 10.1023/B:JOTS.0000048954.50232.d8/full

Beach, S. H. R., Jouriles, E. N., \&O’Leary, K. D. (1985). Extramarital sex: Impact on depression and communication in couples seeking marital therapy. Journal of Sex \&Marital Therapy, 11(2), 99-108. doi: 10.1080/00926238508406075

Bell, K. M, \&Naugle, A. E. (2007). Effects of social desirability on students' self-reporting of partner abuse perpetration and victimization. Violence and Victims. 2(22), 243-256.

Campbell, R., Sullivan, C. M., \&Davidson. W. S. (1995). Women who use domestic violence shelters: Changes in depression over time. Psychology of Women Quartely, 19(2), 237-255. doi: 10.1111/j.1471-6402.1995. tb00290.x

Carlson, B. E., McNutt, L., \&Choi, D. Y. (2003). Childhood and adult abuse among women in primary health care: Effects on mental health. Journal of Interpersonal Violence, 18(8), 924-914. doi: 10.1177/0886260503253882

Clements, P. T., Speck, P. M., Crane, P. A., \&Faulkner, M. J. (2004). Issues and dynamics of sexually assaulted adolescents and their families. International Journal of Mental Health Nursing, 13(4), 267-274. doi: 10.1111/j.1445-8330.2004.00344.x

Coker, A. L., Davis, K. E., Arias, I., Desai, S., Sanderson, M., Brandt, H. M., \& Smith, P. H. (2002). Physical and mental health effects of intimate partner violence for men and women. American Journal of Preventive Medicine,23(4), 260-268. doi: doi: 10.1016/S0749-3797(02)00514-7

Cornelius, T.L., \&Resseguie, N. (2007). Primary and secondary prevention programs for dating violence: A review of the literature. Aggression and Violent Behavior, 12(3), 354-375. doi: 10.1016/j.avb.2006.09.006

Couto, M. T., \& Schraiber, L. B. (2005). Homens, saúde e violência: novas questões de gênero no campo da saúde coletiva. InMinayo, M. C. S, \&Coimbra Junior, C. E. (Orgs.). Críticas e atuantes: Ciências Sociais e Humanas em Saúde na América Latina.(pp. 687-706). Rio de Janeiro: Fiocruz.

DeJonghe, E. S., \&Levendosky, A. A. (2008). Women survivors of intimate partner violence and posttraumatic stress disorder: Prediction and prevention. Journal of Postgraduate Medicine, 54(4), 294-300.

Devries, K., Watts, C. H., Yoshihama, M., Kiss, L. B., Schraiber, L. B, Deyessa, N., ...\& Garcia-Moreno, C. (2011). Violence against women is strongly associated with suicide attempts: Evidence from the WHO multicountry study on women's health and domestic violence against women. Social Science \& Medicine, 73(1),79-86. doi: 10.1016/j.socscimed.2011.05.006

Dye, M. L., \& Eckhardt, C.I. (2000). Anger, irrational beliefs, and dysfunctional attitudes in violent dating relationships. Violence and Victims, 15(3), 337-350.

Eshelman L.,\&Levendosky, A. A. (2012). Dating violence: Mental health consequences based on type of abuse. ViolenceVictims, 27(2), 215-28.

Flake, T.A. (2012). Violência no namoro entre jovens universitários no estado de São Paulo. (Tese de doutorado). Universidade de São Paulo, São Paulo.

Flake, T. B., C., Schraiber, L.B., \& Menezes, P.R. (2013). Violência por parceiro íntimo entre estudantes de duas universidades do Estado de São Paulo, Brasil. Revista Brasileira de Epidemiologia, 16(4), 801-816. doi: 10.1590/ S1415-790X2013000400001

Golding, J. M.(1999). Intimate partner violence as a risk factor for mental disorders: A meta-analysis. Journalof Family Violence, 14(2), 99-132. doi: 10.1023/A:1022079418229

Gomes, R. (2010). A saúde dos homens em foco. São Paulo: Editora UNESP.

Goodkind, J. R., Gillum, T. L., Bybee, D. I, \&Sullivan, C. M. (2003). The impact of family and friends: Reactions on the well-being of women with abusive partners. Violence Against Women, 9(3), 347-373. doi: 10.1177/1077801202250083

Halpern, C. T, Oslak, S. G., Young, M. L., Martin, S. L., \& Kupper, L. L. (2001). Partner violence among adolescents in opposite-sex romantic relationships: Findings from the National Longitudinal Study of Adolescent Health. American Journal of Public Health, 91(10), 1679- 1685. doi: 10.2105/AJPH.91.10.1679

Hamby, S. L.,\&Sugarman, D. B. (1999). Acts of psychological aggression against a partner and their relation to physical assault and gender. JournalofMarriageandthe Family, 61(4), 959-70. 
Howard, D. E, \&Wang, M.Q.(2003). Risk profiles of adolescent girls who were victims of dating violence. Adolescence, 38(149), 1-14.

Hume, M. (2008). The myths of violence: Gender, conflict, and community in El Salvador, Latin American Perspectives, 35(5), 59-76. doi: 10.1177/0094582x08321957

Instituto Nacional de Estudos e Pesquisas Educacionais Anísio Teixeira. (2012). Censo da educação superior: 2010 - resumo técnico, Brasília: Instituto Nacional de Estudos e Pesquisas Educacionais Anísio Teixeira, 2012. Recuperado de <http://download.inep.gov.br/download/ superior/censo/2010/resumo_tecnico_censo_educacao_ superior_2010.pdf>

Jackson, S. M., Cram, F., \& Seymour, F. W. (2000) Violence and sexual coercion in high school students' dating relationships, Journal of Family Violence, 15(1), 23-36. doi: 10.1023/A:1007545302987

Jansen, H. A., Watts, C., Ellsberg, M., Heise, L., \& GarciaMoreno, C. (2004). Interviewer training in the WHO multi-country study on women's health and domestic violence. Violence against women, 10(7), 831-849. doi: 10.1177/1077801204265554

Kaura, S., \&Lohman, B. (2007). Dating violence victimization, relationship satisfaction, mental health problems, and acceptability of violence: A comparison of men and women. Journal of Family Violence, 22(6), 367-381. doi: 10.1007\%2Fs10896-007-9092-0

Knudson-Martin, C., \&Mahoney, A. R. (2009). Couple, Gender, and Power - creating, change intimate relationships. New York: Springer Publishing Company.

Lehrer, J. A., Buka, S., Gortmaker S, \&Shrier, L. A. (2006). Depressive symptomatology as a predictor of exposure to intimate partner violence among US female adolescents and young adults. Archives of Pediatrics \& Adolescent Medicine,160(3), 270-276. doi: 10.1001/ archpedi.160.3.270

McFarlane, J., Malecha, A., Gist, J., Schultz, P., Wilson, P., \&Fredland, N. (2000). Indicators of intimate violence in women's employment: Implications for workplace action. AAOHN Journal, 48(5), 215-220. doi: 10.1177/216507990004800503
Mirowsky, J., \& Ross, C. E. (1989). Psychiatric diagnosis as reified measurement. Journal of Health and Social Behavior,30(1), 11-25.

O'Leary, K.D. (1999). Psychological abuse: A variable deserving attention in domestic violence. Violence and Victims, 14, 3 -23.

Sabina, C., \&Straus, M. A. (2008). Polyvictimization by Dating Partners and Mental Health Among US College Students. Violence and Victims, 23(6) 667-682.

Schraiber, L. B, D’Oliveira, A. F. P. L.,\& Couto, M. T. (2009). Violência e saúde: contribuições teóricas, metodológicas e éticas de estudos da violência contra a mulher. Cadernos de Saúde Pública,25, 205-216.

Schraiber, L. B., \&D’Oliveira, A. F. P. L. (2008). Violência contra mulheres e promoção dos direitos humanos: a contribuição do campo da saúde. Divulgação em Saúde para Debate, (58), 1-10.

Sears, H.A, Byers, E. S., \&Price, E. L. (2007). The co-occurrence of adolescent boys'and girls' use of psychologically, psysically, and sexually abusive behaviours in their dating relationships. Journal of Adolescence, 30(3), 487504. doi: 10.1016/j.adolescence.2006.05.002

Straus, M.A,\&Gelles, R. J. (1990) Physical violence in American families. New Brunswick, NJ: Transaction Publishers.

Straus, M. A. (2004a) Cross-cultural reliability and validity of the Revised Conflict Tactics Scales: A study of university student dating couples in 17 nations. Cross Cultural Research, 38(4), 407-432.

Straus, M. A., Aldrighi, T., Alvarez, S. D., Atan, A., BoeckmannI, Sieber, C., Yodanis, C. L., Bougere, A., Brownridge, D.,... \&Chan, K. L. (2004b). Prevalence of violence against dating partners by male and female university students worldwide. Violence Against Women, 10(7), 790-811.

Sutherland, C. A., Sullivan, C. M., \& Bybee, D. I. (2001). Effects of intimate partner violence versus poverty on women's health. Violence Against Women,7(10), 1122-1143.

Teten, A. L., Ball, B., Valle, L. A., Noonan, R., \& Rosenbluth, B. (2009). Considerations for the Definition, Measurement, Consequences, and Prevention of Dating Violence Victimization among Adolescent Girls. Journal of Women's Health. 18(7), 923-927. doi: 10.1089/jwh.2009.1515 